\title{
Correspondence
}

\section{Re: Effect of Antibiotics on Vulvovaginal Candidiasis: A MetroNet Study}

To the Editor: I read with great interest the recent article by $\mathrm{Xu}$ et al entitled "Effect of Antibiotics on Vulvovaginal Candidiasis" in the July issue of your journal. ${ }^{1}$ In their study they have highlighted a very common etiology for vulvovaginal candidiasis which is becoming all the more common now with widespread overuse of antibiotics. ${ }^{2}$ I understand their study was limited by numbers, but it would be interesting to know if they noted any association with a particular type of antibiotic. Previous studies have implicated certain antibiotics such as cephalosporins $^{3}$ or fluoroquinolones, ${ }^{4}$ whereas others found no relation at all..$^{5}$

$$
\begin{array}{r}
\text { Saira Tandon, MD } \\
\text { ACOG } \\
\text { Brooklyn, NY } \\
\text { dr_saira_ahmad@hotmail.com }
\end{array}
$$

\section{References}

1. Xu J, Schwartz K, Bartoces M, Monsur J, Severson RK, Sobel JD. Effect of antibiotics on vulvovaginal candidiasis: A MetroNet study. J Am Board Fam Med 2008 21:261-8.

2. Xu J, Sobel JD. Antibiotic-associated vulvovaginal candidiasis. Curr Infect Dis Rep 2003;5(6):481-7.

3. MacDonald TM, Beardon PH, McGilchrist MM, Duncan ID, McKendrick AD, McDevitt DG. The risks of symptomatic vaginal candidiasis after oral antibiotic therapy. $\mathrm{Q}$ J Med 1993;86:419-24.

4. Menday AP. Symptomatic vaginal candidiasis after pivmecillinam and norfloxacin treatment of acute uncomplicated lower urinary tract infection. Int J Antimicrob Agents 2002; 20(4):297-300.

5. Spinillo A, Capuzzo E, Acciano S, De Santolo A, Zara F. Effect of antibiotic use on the prevalence of symptomatic vulvovaginal candidiasis. Am J Obstet Gynecol 1999;180: $14-7$.

doi: $10.3122 /$ jabfm.2009.02.080212

\section{Response: Re: Effect of Antibiotics on Vulvovaginal Candidiasis: A Metronet Study}

To the Editor: We appreciate the interest of Dr. Tandon ${ }^{1}$ in our recent paper on the association of antibiotic use with subsequent vulvovaginal candidiasis (VVC). ${ }^{2}$ As we stated in our paper, the small sample size limits our ability to determine the effects of antibiotic type, dosage, or duration on the primary outcomes. In fact, the 6 women who later developed symptomatic VVC were on 6 different antibiotics, and 1 was on 2 antibiotics, with range of antibiotic duration of 5 to 10 days. Because the majority of our study participants had respiratory infections, penicillins (eg, amoxicillin or augmentin) and macrolides (eg, azithromycin) were the most frequently pre- scribed antibiotics, with less usage of quinolones or cephalosporins. Although it is likely that each type of antibiotic has a different effect on the vaginal flora, we were not able to find any distinctive pattern of risk by antibiotic used or the duration of antibiotic therapy. Furthermore, the association of VVC with particular antibiotics has been indirectly indicated by epidemiologic studies $^{3,4}$ rather than directly supported by mycological studies, which are sorely needed.

Jinping $\mathrm{Xu}, \mathrm{MD}$

Wayne State University School of Medicine

Detroit, Michigan

jxu@med.wayne.edu

\section{References}

1. Tandon S. Re: Effect of antibiotics on vulvovaginal candidiasis: A Metronet study. J Am Board Fam Med 2009;22(2):223.

2. Xu J, Schwartz K, Bartoces M, Monsur J, Severson RK, Sobel JD. Effect of antibiotics on vulvovaginal candidiasis: A MetroNet study. J Am Board Fam Med 2008;21(4):261-68.

3. MacDonald TM, Beardon PH, McGilchrist MM, Duncan ID, McKendrick AD, McDevitt DG. The risks of symptomatic vaginal candidiasis after oral antibiotic therapy. Q J Med 1993;86(7):419-24.

4. Menday AP. Symptomatic vaginal candidiasis after pivmecillinam and norfloxacin treatment of acute uncomplicated lower urinary tract infection. Int J Antimicrob Agents 2002; 20(4):297-300

doi: 10.3122/jabfm.2009.02.080258

\section{Re: Predictive Value of Exercise Stress Testing in a Family Medicine Population}

To the Editor: This study adds perspective to the role of exercise stress testing (EST) in triaging primary care patients with cardiac symptoms or risk factors. It also belies its first sentence, "Exercise stress testing (EST) is a screening test for coronary artery disease." 1

Only $2.3 \%$ of the studied patients presented for screening. Another $31 \%$ of patients received EST for no listed indication or for pre-exercise evaluation. Though the retrospective study design limits our insight, it is more likely than not that these additional patients had cardiac symptoms or risk factors.

In this study, all patients with positive cardiac outcomes, regardless of EST results, had at least 1 risk factor. All asymptomatic patients had a very low or low pretest probability of coronary artery disease, with associated high negative predictive values (NPVs) of EST. Unless triage of at-risk (by symptoms or risk factors) primary care patients is conflated with screening (attempts to detect treatable presymptomatic disease), this combination of findings affirms both the effectiveness of 